\title{
Severe ineffective erythropoiesis discriminates prognosis in myelodysplastic syndromes: analysis based on 776 patients from a single centre
}

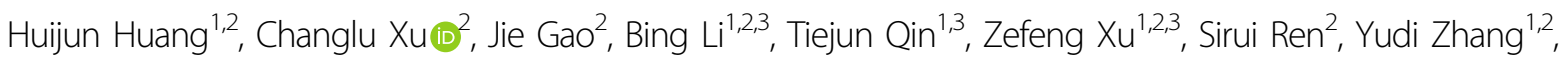

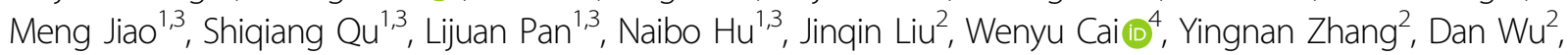

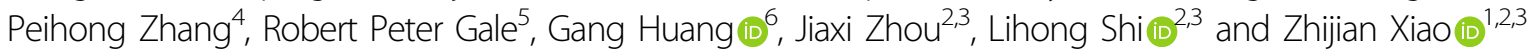

\begin{abstract}
The underlying mechanisms and clinical significance of ineffective erythropoiesis in myelodysplastic syndromes (MDS) remain to be fully defined. We conducted the ex vivo erythroid differentiation of megakaryocytic-erythroid progenitors (MEPs) from MDS patients and discovered that patient-derived erythroblasts exhibit precocity and premature aging phenotypes, partially by inducing the pro-aging genes, like ERCC1. Absolute reticulocyte count (ARC) was chosen as a biomarker to evaluate the severity of ineffective erythropoiesis in 776 MDS patients. We found that patients with severe ineffective erythropoiesis displaying lower ARC $\left(<20 \times 10^{9} / L\right)$, were more likely to harbor complex karyotypes and high-risk somatic mutations $(p<0.05)$. Lower ARCs are associated with shorter overall survival $(O S)$ in univariate analysis $(p<0.001)$ and remain significant in multivariable analysis. Regardless of patients of lower-risk who received immunosuppressive therapy or higher-risk who received decitabine treatment, patients with lower ARC had shorter OS $(p<0.001)$. Whereas no difference in OS was found between patients receiving allo-hematopoietic stem cell transplantations (Allo-HSCT) $(p=0.525)$. Our study revealed that ineffective erythropoiesis in MDS may be partially caused by premature aging and apoptosis during erythroid differentiation. MDS patients with severe ineffective erythropoiesis have significant shorter OS treated with immunosuppressive or hypo-methylating agents, but may benefit from Allo-HSCT.
\end{abstract}

\section{Introduction}

Myelodysplastic syndromes (MDS), a heterogeneous clonal myeloid neoplasm characterized by ineffective hematopoiesis leading to cytopenia(s) and a risk of leukemia transformation ${ }^{1}$, is one of the most frequently encountered acquired bone marrow failure (BMF)

Correspondence: Lihong Shi (shilihongxys@ihcams.ac.cn) or

Zhijian Xiao (zjxiao@ihcams.ac.cn)

${ }^{1}$ MDS and MPN Centre, Institute of Hematology and Blood Diseases Hospital, Chinese Academy of Medical Sciences \& Peking Union Medical College, Tianjin, China

${ }^{2}$ State Key Laboratory of Experimental Hematology, Institute of Hematology and Blood Diseases Hospital, Chinese Academy of Medical Sciences \& Peking Union Medical College, Tianjin, China

Full list of author information is available at the end of the article

These authors contributed equally: Huijun Huang, Changlu Xu, Jie Gao syndromes in adults. Genetic and epigenetic changes that affect hematopoietic stem cells (HSCs) and alterations in the hematopoietic niche resulting in degeneration and apoptosis in hematopoietic stem and progenitor cells (HSPCs) mainly contribute to ineffective hematopoiesis ${ }^{2,3}$.

Ineffective erythropoiesis refers to the inability to produce adequate red blood cells (RBCs) with increased dysplastic erythroid precursors in the BM. Anemia is the most common symptom in MDS which correlates with ineffective erythropoiesis. Previous studies suggested that the deficiencies of erythroid lineage of MDS patients were more pronounced than other myeloid lineages ${ }^{4}$, highlighting the widespread ineffective erythropoiesis in MDS. Ineffective erythropoiesis in MDS has long been attributed to the differentiation arrest and increased apoptosis

\section{(c) The Author(s) 2020}

(c) (i) Open Access This article is licensed under a Creative Commons Attribution 4.0 International License, which permits use, sharing, adaptation, distribution and reproduction cc) in any medium or format, as long as you give appropriate credit to the original author(s) and the source, provide a link to the Creative Commons license, and indicate if changes were made. The images or other third party material in this article are included in the article's Creative Commons license, unless indicated otherwise in a credit line to the material. If material is not included in the article's Creative Commons license and your intended use is not permitted by statutory regulation or exceeds the permitted use, you will need to obtain permission directly from the copyright holder. To view a copy of this license, visit http://creativecommons.org/licenses/by/4.0/. 
of erythroid precursors induced by different mechanisms, such as genetic lesions originating in HSCs, excessive proinflammatory cytokines and immune disorders ${ }^{3,5-7}$. Recent investigations also implicated activation of the NLRP3 inflammasome in HSPCs as a critical convergent signal in MDS with consequential pyroptotic cell death ${ }^{8,9}$. However, other potential mechanisms responsible for defective erythropoiesis are still elusive. Moreover, a reliable biomarker to accurately reflect the severity of BM ineffective erythropoiesis has been lacking so far and its prognostic significance remains to be carefully defined.

In this study, the HSCs and megakaryocytic-erythroid progenitors (MEPs) in the BM mononuclear cells (BMMNCs) of MDS patients $(n=31)$ were measured by flow cytometry and in vitro colony-forming unit cell (CFU-C) were assayed in 612 MDS patients, and we found that the percentage of HSCs and MEPs in the BMMNCs, the numbers of burst-forming unit-erythroid (BFU-E) and colony forming unit-erythroid (CFU-E) of MDS patients were sifgnificantly reduced compared with health controls. The ex vivo erythroid differentiation of MEPs derived from MDS patients $(n=31)$ were conducted and we found the occurrence of precocity in erythroid progenitor and precursor cells of MDS patients which were prone to undergo subsequent premature aging and apoptosis. As the absolute reticulocyte count (ARC) can reflect the erythropoietic activity of $\mathrm{BM}$ properly ${ }^{10}$, we therefore chose ARC as a biomarker to evaluate the severity of ineffective erythropoiesis. Here, we adopted a cut-off of ARC less than $20 \times 10^{9} / \mathrm{L}$, which is one of the diagnostic criteria of severe aplastic anemia (SAA) in guidelines recommended by the British Committee for Standards in Haematology $(\mathrm{BCSH})^{11}$, to discriminate the extent of ineffective erythropoiesis. Based on this, a cohort of 776 newly diagnosed MDS patients in our center were divided into two groups and their clinical outcomes were investigated. Our data showed that a severe reduced ARC $\left(<20 \times 10^{9} / \mathrm{L}\right)$ acted as a powerful independent prognostic factor in MDS which could not be reversed by immunosuppressive treatments in Revised International Prognostic Scoring System (IPSS-R) lower-risk patients and hypo-methylating agents in IPSS-R higher-risk patients, but had no impact on the survival of patients in both IPSS-R categories when received allo-hematopoietic stem cell transplantation (Allo-HSCT).

\section{Methods}

\section{Patients and samples}

BM samples and umbilical cord blood were harvested after informed consent approved by the Ethical Committee on Medical Research at Institute of Hematology and blood disease hospital. In detail, BM samples were collected from 31 newly diagnosed MDS patients according to the 2016 revised criteria of the World Health
Organization $(\mathrm{WHO})^{12}$, and 12 healthy donors for the cellular studies and transcriptome sequencing analysis. Clinical features of these patients are shown in Table S1. Additionally, a total of 776 consecutive newly diagnosed MDS patients at our center, from December 2011 to December 2018, constituted the clinical cohort. BM and Blood samples were obtained from all patients at diagnosis. Wright-Giemsa-stained BM and Blood smears were reviewed according to the 2016 revised criteria by two expert pathologists (W Cai, P Zhang). Detailed characteristics of all patients are shown in Table 1. A total of $678(87.4 \%)$ patients had evaluable karyotypes. 612 (78.9\%) patients experienced CFU-C assays, the reference of normal average (Mean $\pm \mathrm{SD}$ ) at our lab were: BFU-E $31 \pm 6$, CFU-E $81 \pm 12$ and colony forming unit-granulocyte/macrophage (CFU-G/M) $22 \pm 7^{13} .553$ patients were tested for serum erythropoietin (EPO) levels at diagnosis. DNA derived from BMMNCs from all patients underwent the targeted 112-gene sequencing at diagnosis ${ }^{14}$. The prognostic categories were evaluated with the IPSS- $\mathrm{R}^{15}$. An IPSS-R scores $\leq 3.5$ were classified into a lower-risk cohort while $>3.5$ into a higher-risk cohort ${ }^{16}$. Data of treatments were available for 575 patients. Among them, 48 (8.3\%) received erythropoietin with or without G-CSF, RBC and/or platelet transfusions; 303 (52.7\%) patients received immunosuppressive drugs using cyclosporine combined with danazol ( \pm thalidomide); 91 (15.8\%) received decitabine; 38 (6.6\%) received chemotherapy using aclacinomycin or homoharringtonine combined with cytarabine and granulocyte-colony stimulating factor (G-CSF; termed CAG or HAG regimen), idarubicin or daunorubicin combined with cytarabine (IA or DA) or melphalan; 74 (12.9\%) received Allo-HSCT, and 21 (3.7\%) received traditional Chinese medicines. Follow-up data were available for $687(88.5 \%)$ patients with a median follow-up of 17 months (range, 2-87 months). Overall survival (OS) was calculated from the date of diagnosis to the date of last follow-up or death. All subjects provided informed consent in compliance with the Declaration of Helsinki.

\section{Fluorescence-activated cell sorting analysis}

The procedure for Fluorescence-activated cell sorting (FACS) staining was performed as described previously ${ }^{17}$. For detailed information of sorting strategy of HSCs and MEPs, please refer to supplementary methods.

\section{Ex vivo erythroid differentiation}

Purification of $\mathrm{CD}_{3} 4^{+}$cells from BM of MDS patients and healthy donors or from human umbilical cord blood was carried out as previously ${ }^{18}$. The purified CD34 ${ }^{+}$cells or FACS sorted MEPs were induced ex vivo differentiation toward erythroid lineage as previously described ${ }^{18}$. Please refer to Supplementary Methods for details. 
Table 1 Clinical and laboratory characteristics of 776 patients with MDS.

\begin{tabular}{|c|c|c|c|c|}
\hline Characteristics & ARC $<20 \times 10^{9} / \mathrm{L}(N=169)$ & $\mathrm{ARC} \geq 20 \times 10^{9} / \mathrm{L}(N=607)$ & Total $(N=776)$ & $p$ value \\
\hline Sex, $n(\%)$ & & & & 0.046 \\
\hline Male & 119 (70.4\%) & 375 (61.8\%) & 494 (63.7\%) & \\
\hline Female & $50(29.6 \%)$ & 232 (38.2\%) & $282(36.35 \%)$ & \\
\hline Age, median (range), y & $56(16-83)$ & $52(14-83)$ & $54(14-83)$ & 0.032 \\
\hline Age $\geq 60$ years, $n(\%)$ & $73(43.2 \%)$ & 199 (32.8\%) & $272(35.1 \%)$ & 0.014 \\
\hline WHO classification 2016, n (\%) & & & & 0.101 \\
\hline MDS-SLD & $4(2.4 \%)$ & $30(4.9 \%)$ & $34(4.4 \%)$ & \\
\hline MDS-RS-SLD & $4(2.4 \%)$ & $18(3.0 \%)$ & $22(2.8 \%)$ & \\
\hline MDS-MLD & $71(42.0 \%)$ & $307(50.6 \%)$ & $378(48.7 \%)$ & \\
\hline MDS-RS-MLD & $5(3.0 \%)$ & $10(1.6 \%)$ & $15(1.9 \%)$ & \\
\hline MDS-EB1 & $39(23.1 \%)$ & $114(18.8 \%)$ & $153(19.7 \%)$ & \\
\hline MDS-EB2 & $41(24.3 \%)$ & $104(17.1 \%)$ & 145 (18.7\%) & \\
\hline MDS with isolated del (5q) & 0 & $7(1.2 \%)$ & $7(0.9 \%)$ & \\
\hline MDS-U & $5(3.0 \%)$ & $17(2.8 \%)$ & $22(2.8 \%)$ & \\
\hline $\mathrm{Hb}$, median (range), g/L & $64(31-138)$ & $83(38-155)$ & $78(31-155)$ & $<0.001$ \\
\hline WBC, median (range), $\times 10^{9} / \mathrm{L}$ & $2.51(0.71-21.17)$ & $2.83(0.61-20.42)$ & $2.75(0.61-21.17)$ & 0.007 \\
\hline ANC, median (range), $\times 10^{9} / \mathrm{L}$ & $0.99(0.04-13.18)$ & $1.19(0-17.37)$ & $1.15(0-17.37)$ & 0.002 \\
\hline PLT, median (range), $\times 10^{9} / \mathrm{L}$ & $51(2-536)$ & $63(2-694)$ & $60(2-694)$ & 0.012 \\
\hline BM erythroblasts, median (range), \% & $20(0-75)$ & $35(0-92.5)$ & $31(0-92.5)$ & $<0.001$ \\
\hline $\begin{array}{l}\text { Sum of proerythroblast and basophilic erythroblast(E1), } \\
\text { median (range), \% }\end{array}$ & $1(0-30)$ & $1.5(0-18.5)$ & $1.5(0-30)$ & 0.009 \\
\hline $\begin{array}{l}\text { Sum of polychromatic and orthochromatic erythroblast (E2), } \\
\text { median (range), \% }\end{array}$ & $17.5(0-67)$ & $32(0-88)$ & $29(0-88)$ & $<0.001$ \\
\hline Ratio of E1 to E2, median (range) & $0.052(0-1.75)$ & $0.05(0-2)$ & $0.05(0-2)$ & 0.305 \\
\hline BM blast, median (range), \% & $3.5(0-19.5)$ & $2.5(0-19.5)$ & $2.5(0-19.5)$ & 0.005 \\
\hline IPSS-R karyotype, $n(\%), N=678$ & & & & $<0.001$ \\
\hline Very good & $1(0.7 \%)$ & $7(1.3 \%)$ & $8(1.2 \%)$ & \\
\hline Good & $69(48.6 \%)$ & 313 (58.4\%) & $382(56.3 \%)$ & \\
\hline Intermediate & $26(18.3 \%)$ & $138(25.7 \%)$ & $164(24.2 \%)$ & \\
\hline Poor & $11(7.7 \%)$ & $29(5.4 \%)$ & $40(5.9 \%)$ & \\
\hline Very poor & $35(24.6 \%)$ & 49 (9.1\%) & $84(12.4 \%)$ & \\
\hline Complex karyotype, $n(\%)$ & $39(27.5 \%)$ & $68(12.7 \%)$ & $107(15.8 \%)$ & $<0.001$ \\
\hline IPSS-R risk group, $n(\%), N=678$ & & & & $<0.001$ \\
\hline Very low & 0 & $21(3.9 \%)$ & $21(3.1 \%)$ & \\
\hline Low & $21(14.8 \%)$ & $148(27.6 \%)$ & 169 (24.9\%) & \\
\hline Intermediate & $41(28.9 \%)$ & $175(32.6 \%)$ & $216(31.9 \%)$ & \\
\hline High & $30(21.1 \%)$ & $122(22.8 \%)$ & $152(22.4 \%)$ & \\
\hline Very high & $50(35.2 \%)$ & $70(13.1 \%)$ & $120(17.7 \%)$ & \\
\hline IPSS-R two groups, $n$ (\%) & & & & $<0.001$ \\
\hline Lower-risk & $40(28.2 \%)$ & $245(45.7 \%)$ & $285(42.0 \%)$ & \\
\hline Higher-risk & $102(71.8 \%)$ & 291 (54.3\%) & 393 (58.0\%) & \\
\hline
\end{tabular}

MDS myelodysplastic syndrome, $A R C$ absolute reticulocyte count, MDS-SLD MDS with single lineage dysplasia, MDS-RS-SLD MDS with ring sideroblasts with single lineage dysplasia, MDS-MLD MDS with multilineage dysplasia, MDS-RS-MLD MDS with ring sideroblasts with multilineage dysplasia, MDS-EB1 MDS with excess blasts-1, MDS-EB2 MDS with excess blasts-2, MDS-U MDS unclassifiable, $H b$ haemoglobin, WBC white blood coun, ANC absolute neutrophil count, PLT platelet count, BM bone marrow, IPSS-R Revised International Prognostic Scoring System.

\section{T and K562 cell culture}

293T and K562 cells were obtained from the American Type Culture Collection (ATCC) and cultured according to ATCC recommendations. Erythroid differentiation of K562 cells was induced with hemin $(30 \mu \mathrm{M})$ for 3 days.

\section{Wright-Giemsa and benzidine staining}

Cell morphology was analyzed by Wright-Giemsa staining (Baso, Cat\# BA-4017). Hemoglobin synthesis was assessed by benzidine staining ${ }^{19}$. The benzidinepositive cells were stained in brown.

\section{Measurement of cellular aging}

Senescent cell detecting was performed using a $\beta$-galactosidase staining Kit (Beyotime biotechnology), according to the manufacturer's instructions. Senescent cells will be stained blue.

\section{RNA-Seq and Gene sets enrichment analysis}

Library construction and data processing were performed by Novogene (Beijing, China) as previously described $^{18}$. The gene expression matrix was used to conduct Gene sets enrichment analysis (GSEA). RNA 
sequencing data has been uploaded to the Gene Expression Omnibus database. If there is any reasonable need, please contact the corresponding author for the accession number. Detailed information were described in supplementary methods.

\section{RNA extraction and qRT-PCR}

Total RNA extraction and quantitative real-time PCR (qRT-PCR) were performed as mentioned before ${ }^{18}$. Primer sequences used for qRT-PCR are shown in Table S2.

Plasmid construction, lentivirus production, and infection

The short hairpin RNA (shRNA) sequences targeting $E R C C 1$ were cloned into lentiviral shRNA expression vector pSIH-H1, containing a GFP expression marker. Lentiviruses were packaged using ViraPower Lentiviral Packaging system (Invitrogen). For infection, K562 cells and primary erythroid cells at day 4 of differentiation were incubated with lentiviruses for $12 \mathrm{~h}$ before washing away the excess virus. The shRNA sequences are listed in Table S2.

\section{Western blotting}

Detailed information for western blotting (WB) were described before ${ }^{18}$. Specially, antibody to ERCC1 (Proteintech) was diluted to a final concentration of 1:300 and $\alpha$-Tubulin (Abcam) was diluted to 1:5000, which served as the loading control.

\section{Statistical analysis}

Categorical variables were analyzed by the Fisher test and continuous variables by Mann-Whitney $U$ test or Kruskal-Wallis analysis, as appropriate. Survival analysis was carried out with the Kaplan-Meier method and compared with the log-rank test. Cox proportional hazard regression model was used for multivariable analysis. Two-tailed $p$ values $<0.05$ were considered significant. All the analyses were conducted using SPSS, version 25.0.

\section{Results}

Erythropoietic activity was impaired in BM of MDS patients

The HSCs and MEPs in the BMMNCs of MDS patients $(n=31)$ and healthy donors $(n=12)$ were measured by flow cytometry with the markers of $\mathrm{Lin}^{-} \mathrm{CD} 34^{+} \mathrm{CD} 38^{-} \mathrm{C}$ $\mathrm{D}_{45 \mathrm{RACD}^{-} \mathrm{CD}} 3^{-}$for $\mathrm{HSC}$ and $\mathrm{Lin}^{-} \mathrm{CD} 34^{+} \mathrm{CD} 38^{+} \mathrm{C}-$ D45RA $^{-}$CD123 ${ }^{-}$for MEP (Fig. S1) ${ }^{17}$. Both percentage of HSCs and MEPs in Lin ${ }^{-}$cells were lower in MDS patients compared with healthy controls $(p=0.001 ; p=0.084$; Fig. S2A, B). As a consequence of the shortage of HSPCs, we observed the low production of erythroid progenitors, including BFU-E and CFU-E (Fig. S2C). Next, to investigate the capacity of terminal erythroid differentiation of MDS patients, we analyzed the percentage of pro-, basophilic, polychromatic and orthochromatic erythroblasts in BM of 776 MDS patients, respectively. The median BM erythroblast percentage was $31 \%(0-92.5 \%$, Table 1), of which the frequency of relative immature proerythroblast and basophilic erythroblast (E1) comprised 1.5\% (0-30\%, Table 1), and the more differentiated polychromatic and orthochromatic erythroblast (E2) constituted 29\% (0-88\%, Table 1). The median ratio of E1 to E2 was 0.05 (0-2, Table 1), which was higher than that in chronic aplastic anemia patients reported in our previous study ${ }^{20}$, revealing the blockage of erythroid differentiation to a certain extent in MDS. Collectively, BM erythropoietic activity was impaired in MDS patients due to both quantitative and qualitative defects of erythroid precursors.

\section{Precocity and premature aging occurring in patient- derived erythroid cells may be involved in ineffective erythropoiesis}

To elucidate the underlying mechanism of the ineffective erythropoiesis, MEPs from MDS patients $(n=31)$ and healthy donors $(n=12)$ were purified and induced differentiation towards erythroid lineage ex vivo for 18 days. As shown in Fig. 1a, during the process of differentiation, compared to the regular cell morphology, we observed the aberrant differentiated cells with vacuolate cytoplasm and deformed cell membranes in MDS patients on day 4 of differentiation. The synthesis of hemoglobin is vital for functional maturity of erythroid cells. Here, we conducted the Benzidine staining to monitor globin synthesis in patient-derived erythroid cells at various differentiation stages. Intriguingly, we detected the benzidine-positive cells (brown, indicated by arrow, 4.5\%) as early as day 4 of differentiation in cells from patients, when the majority of cells in normal controls are lineage progenitors and incapable of producing hemoglobin (Fig. 1a). Such a trend of more active hemoglobin synthesis in MDS-derived cells persisted throughout the differentiation ( 44 vs. $26 \%$ at day 11 and 54 vs. $40 \%$ at day 14 ) except the final stage (day 18 ) (Fig. S3A, B), despite they did not reach the statistical significance $(p>0.05)$ probably due to the limited sample size. These results suggested that precocity, especially from a functional perspective, may occur during erythroid differentiation in MDS patients. We next utilized senescence-associated $\beta$-galactosidase staining, by which the aging cells could be stained blue. We observed that on average $43.2 \%$ of patient-derived cells from day 8 of culture were dyed blue, which was much higher than that of healthy donors $(43.2$ vs. $10 \%, p=0.007$; Fig. $1 \mathrm{a}$ and Fig. S3C). This result confirmed a premature aging of erythroid precursors in MDS-derived cells. Inherited with such intrinsic disorders, the cell proliferation curve, which was drawn according to the numbers of cells at each stage, showed that the proliferation ability of erythroid cells derived from MEPs of MDS patients was significantly decreased when compared with normal controls (Fig. 1b). 


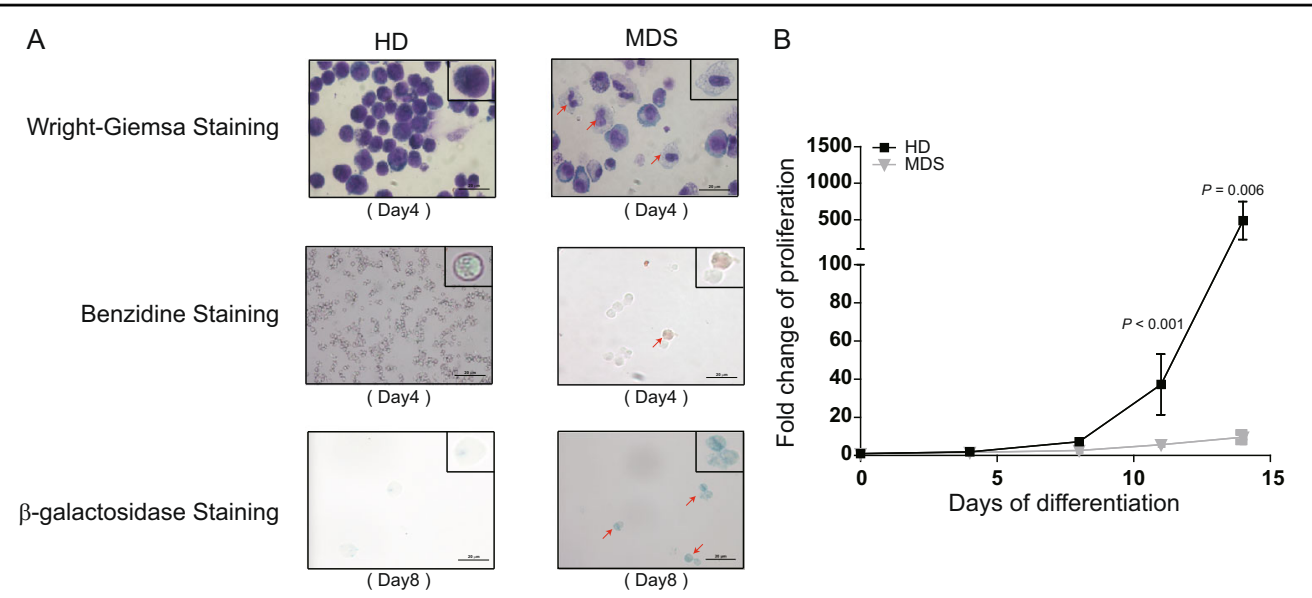

Fig. 1 Inducing ex vivo erythroid lineage differentiation from MDS patients derived megakaryocytic-erythroid progenitors (MEPs). a Representative cytospin staining images of erythroid cells derived from MDS patients and healthy donors (HD) cultured for 4 days and 8 days. The cells indicated by red arrows refer to dysplastic erythrocytes, benzidine-positive and $\beta$-galactosidase-positive cells, respectively, in MDS patients which suggested the precocity and prematuring aging in erythroid differentiation of MDS. Scale bar $=20 \mu \mathrm{m}$. $\mathbf{b}$ Cell proliferation curve was drawn according to cell counts at each stage.

The aberrant molecular pathways account for the premature aging of erythroid cells from MDS patients

To further explore the molecular mechanisms of the ineffective erythropoiesis in MDS, we next conducted low-input RNA-seq analysis in highly purified HSCs (20-200 cells), MEPs (20-200 cells) and cultured erythroid cells at day 4 and 8 of differentiation from purified MEPs. GSEA analysis showed that several signaling pathways were significantly different between MDS patients and healthy donors (Fig. S4).

To further provide the molecular evidence of premature aging in MDS patients, we particularly examined the dynamic alteration of gene expression associated with aging. As shown in Fig. 2a, the aging pathway was gradually upregulated in MDS patients from HSCs to erythroid cells at day 8, which was in line with the quantitative analysis (Fig. 2b). The aberrant expression of aging-associated genes in patients was identified as well (Fig. S5). For instance, we detected ERCC1, which is proved to be related to glomerular aging and Parkinson's disease $^{21,22}$; EIF2S1, which is the target gene of Nuclear respiratory factor 1 (NRF1). NRF1 is a transcription factor that activates the expression of a wide range of genes associated with neurodegenerative diseases ${ }^{23}$; and SRR, which is one of single-nucleotide polymorphisms (SNPs), may contribute to age-related cognitive decline ${ }^{24}$. Among them, the trend of higher expression of ERCC1 was further confirmed in MDS-derived erythroid cells during ex vivo differentiation by qRT-PCR (Fig. 2c). Additionally, consistent with previous studies, we also detected the active apoptotic signaling pathway in MDS patients (Fig. 2a) $)^{25,26}$.
To unravel the role of ERCC1 during hematopoietic aging, we infected lentiviral-mediated ERCC1-targeting shRNAs into K562 cells or primary erythroid cells differentiated for 4 days from cord blood CD $34^{+}$cells. The reduced expression of ERCC1 was confirmed on mRNA level by qRT-PCR and protein level by WB in both primary erythroid cells and K562 cells, respectively (Figs. 2d and S6A). In ERCC1 diminished cells, we found that there were much less $\beta$-galactosidase positive cells in primary erythroid cells at day 8 of differentiation (Fig. 2e, f), which was in line with hemin-induced K562 cells (Fig. S6B, C). Collectively, these results imply ERCC1 promotes the aging process of erythroid cells. Additionally, as the result of its pro-aging effects, we also found ERCC1 deficient K562 cells showed stronger proliferation ability compared with scramble control during hemin-induced erythroid differentiation (Fig. S6D). Therefore, the induced ERCC1 expression might reflect the premature aging of the MDSderived erythroid precursors.

\section{Severe reduced ARC is strongly correlated with high-risk cytogenetic and molecular genetic aberrations}

The main feature of MDS is stem-cell-derived clonal myelopoiesis with altered proliferation and differentiation leading to dyshematopoiesis. Genetic defects play a fundamental role in pathogenesis of $\mathrm{MDS}^{27}$. To determine the association between genetic aberrations and dyserythropoiesis, we analyzed results of chromosome abnormalities and somatic gene mutations in 776 MDS patients stratified by levels of ARC given that ARC serves as an indicator reflecting $\mathrm{BM}$ erythropoietic activity. Overall, the median ARC level of all patients was 43.1 


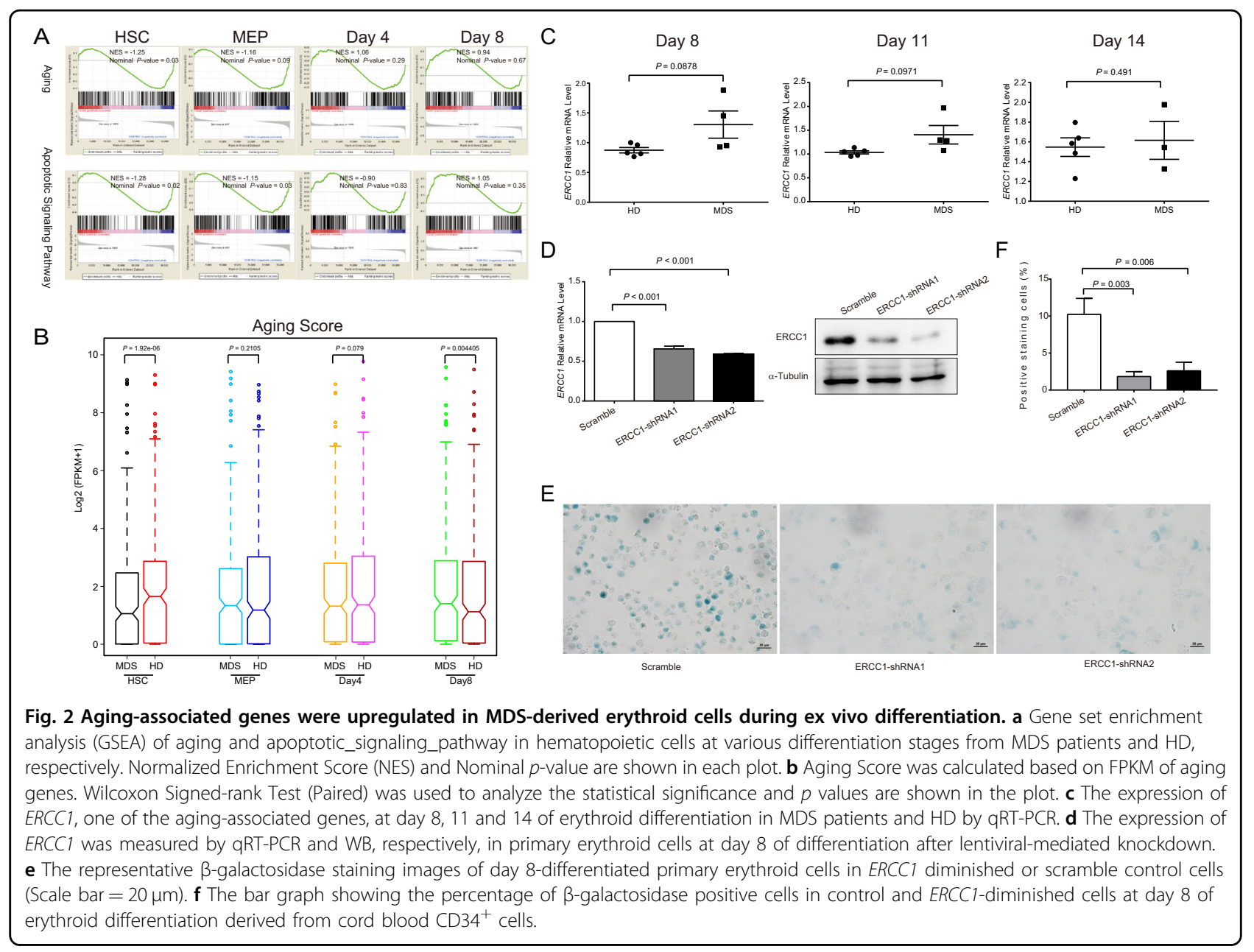

$(0.8-660) \times 10^{9} / \mathrm{L}$. Distributions of ARC among different WHO diagnostic categories and IPSS-R risk groups are shown in Fig. S7. The 776 patients were divided into two cohorts: lower ARC $\left(<20 \times 10^{9} / \mathrm{L}, n=169\right)$ and higher ARC $\left(\geq 20 \times 10^{9} / \mathrm{L}, n=607\right)$ cohorts. Patients in the lower ARC cohort had lower hemoglobin levels $(64 \mathrm{~g} / \mathrm{L}$ vs. $83 \mathrm{~g} /$ $\mathrm{L} ; p<0.001$; Table 1), lower white blood cell (WBC) count $\left(2.51 \times 10^{9} / \mathrm{L}\right.$ vs. $2.83 \times 10^{9} / \mathrm{L} ; p=0.007$; Table 1$)$, lower neutrophil absolute count $\left(0.99 \times 10^{9} / \mathrm{L}\right.$ vs. $1.19 \times 10^{9} / \mathrm{L}$; $p=0.002$; Table 1$)$, lower platelet count $\left(51 \times 10^{9} / \mathrm{L}\right.$ vs. $63 \times 10^{9} / \mathrm{L} ; p=0.012$; Table 1$)$, lower $\mathrm{BM}$ erythroblast percentage (20\% vs. $35 \%$; $p<0.001$; Table 1; Fig. S8A) but a higher $\mathrm{BM}$ blast percentage $(3.5 \%$ vs. $2.5 \% ; p=0.005$; Table 1) than patients in higher ARC cohort. CFU-C assays further confirmed that patients with lower ARC had fewer numbers of CFU-E (30 vs. 40/10 $0^{5}$ BMMNCs; $p=0.004$; Fig. S8B), BFU-E (12 vs. 20/10 5 BMMNCs; $p=$ 0.001; Fig. S8B) and CFU-GM (10 vs. $12 / 10^{5}$ BMMNCs; $p=0.041$; Fig. S8B). Moreover, patients with lower ARC more frequently had an EPO level greater than $500 \mathrm{mIU} /$ $\mathrm{mL}(55.7 \%$ vs. $22.9 \% ; p<0.001$; Fig. S8C), which may arise from the compensatory EPO secretion from kidney due to the severe ineffective erythropoiesis in lower ARC patients. All these data evidenced that patients with lower ARC had more severe ineffective hematopoiesis, especially erythropoiesis.

We noticed that patients in the lower ARC cohort who had severe impaired erythropoiesis were more likely to have complex karyotypes compared with patients in the higher ARC cohort (27.5\% vs. $12.7 \%$; $p<0.001$; Fig. 3a). Additionally, we sequenced 112 frequently mutant genes in MDS in diagnostic BM samples from all patients and identified 1470 high-confidence mutations. In total, 643 of 776 patients $(82.9 \%)$ harbored at least 1 mutation with a median of $2(0-8)$ mutations per sample. The eight most frequently mutated genes in the total cohort were U2AF1 (20.2\%), ASXL1 (12.2\%), SF3B1 (9.4\%), TP53 (7.3\%), RUNX1 (7.1\%), DNMT3A (6.7\%), TET2 (6.3\%) and SETBP1 (5.8\%). Distributions of mutations with a variable allele frequency $>2 \%$ in the lower and higher ARC cohorts are shown in Fig. 3b. Importantly, ZRSR2, TET2, TP53, $N P M 1$, and $M P L$ were mutated more frequently in 

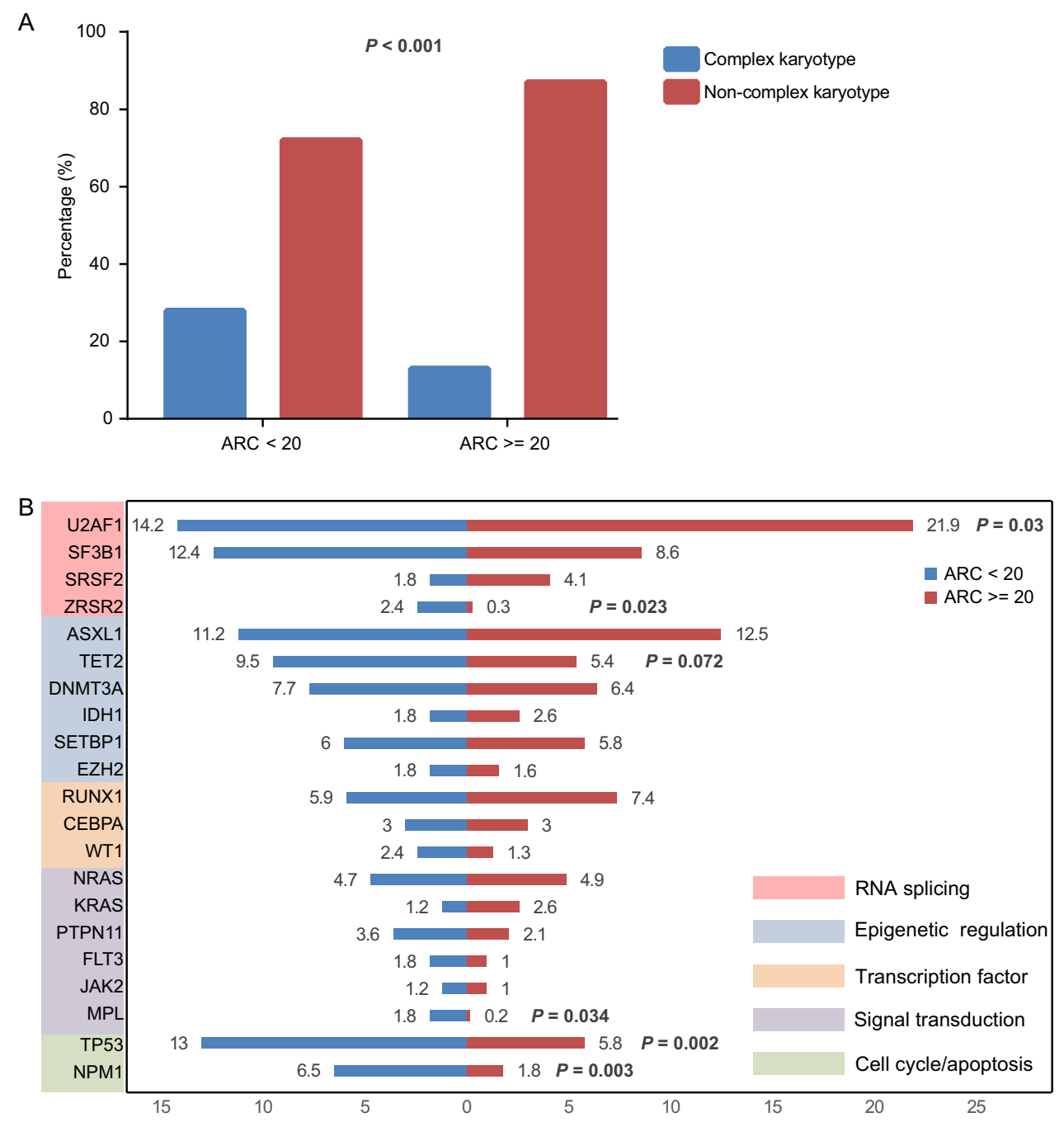

Fig. 3 Associations between ineffective erythropoiesis and genetic abnormalities. a The percentage of complex karyotype in patients with different degree of ineffective erythropoiesis (stratified by lower and higher ARC). $\mathbf{b}$ The genetic distributions in MDS patients with lower and higher ARC.

patients with lower ARC $(p=0.023 ; p=0.072 ; p=0.002$; $p=0.003 ; p=0.034$; Fig. $3 \mathrm{~b}$ ), whereas $U 2 A F 1$ mutations were enriched in higher ARC patients ( $p=0.03$; Fig. $3 \mathrm{~b})$.

\section{Severe reduced ARC is a powerful independent prognostic factor of OS in MDS patients}

In total cohort, median OS of patients with lower ARC was 14 months $(95 \%$ confidence interval [CI], 12-16 months), which was significantly shorter than that of patients with higher ARC (48, 95\% CI, 31-65 months; $p<0.001$; Fig. 4a). In univariate analysis, besides ARC $<$ $20 \times 10^{9} / \mathrm{L}(\mathrm{HR}=2.685,95 \% \mathrm{CI}, 2.045-3.525, p<0.001)$, age ( $\geq 60$ years), IPSS-R higher-risk group and several gene mutations showed significant associations with inferior OS $(p<0.05$; Table 2$)$.
Next, to determine the relative contribution of clinical and genetic factors to OS, we generated a multivariable Cox model including variables related to poor prognosis in univariate analysis $(p<0.1)$. We found that ARC $<20 \times$ $10^{9} / \mathrm{L}$ retained its significance in OS after adjusting for mutation status and other clinical variables $(\mathrm{HR}=2.118$, 95\% CI, 1.563-2.870, $p<0.001)$. Other independent prognostic variables for poor OS were age ( $\geq 60$ years), higher-risk group in IPSS-R and mutated SETBP1 and PTPN11 (Table 2).

Patients with lower ARC in all IPSS-R risk groups except high-risk group had shorter OS compared with patients with higher ARC (Fig. S9). Analysis of very low-risk group were missing due to all patients in this group had an ARC greater than $20 \times 10^{9} / \mathrm{L}$. Both in IPSS-R lower and higher 


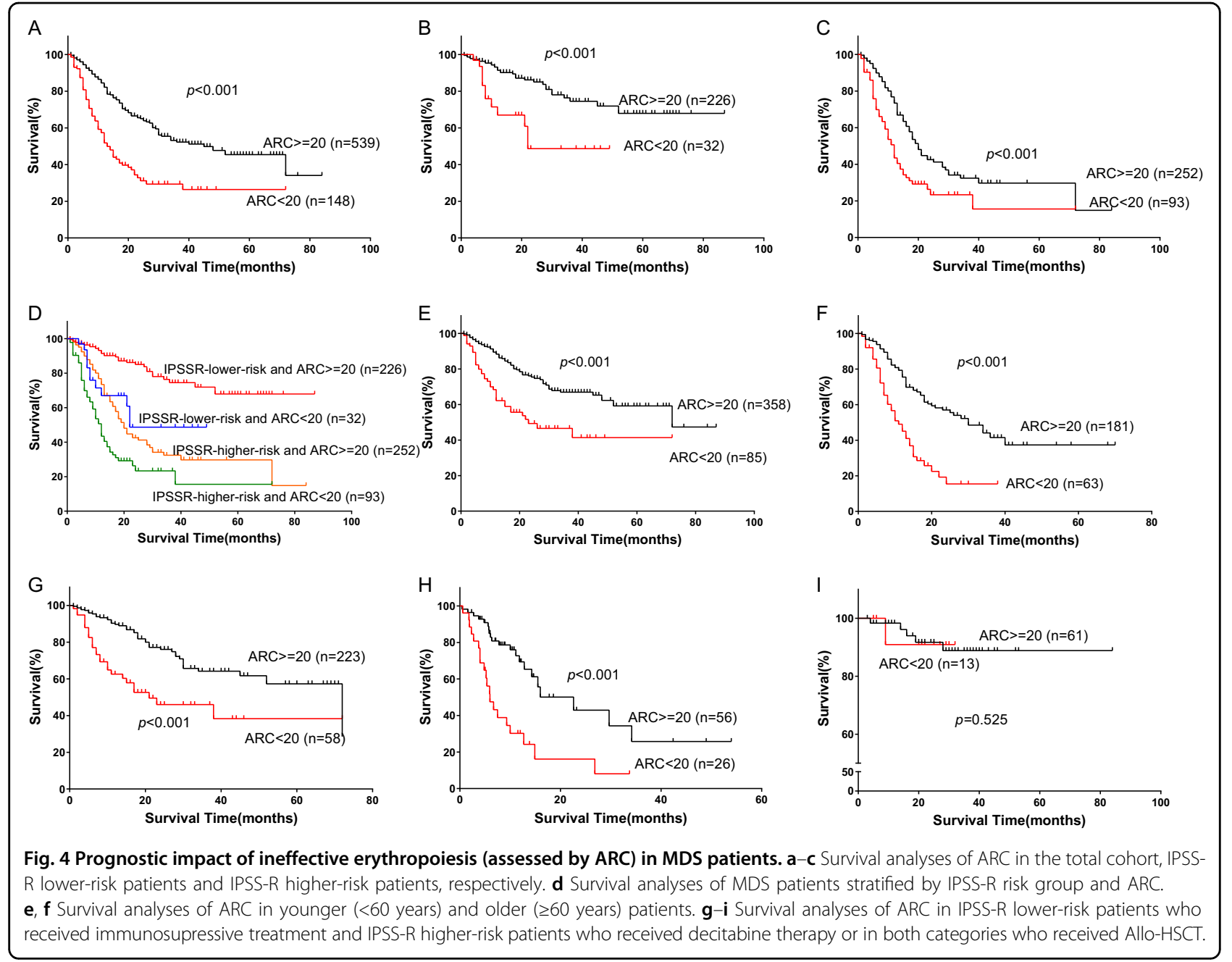

Table 2 Univariate and multivariate analysis of overall survival in the total cohort.

\begin{tabular}{|c|c|c|c|c|c|c|c|c|c|}
\hline \multirow[t]{2}{*}{ Variables } & \multicolumn{3}{|c|}{ Univariate analysis } & \multicolumn{3}{|c|}{ Adjusted by molecular profiles } & \multicolumn{3}{|c|}{ All-inclusive multivariate analysis } \\
\hline & HR & $95 \% \mathrm{Cl}$ & $p$ value & HR & $95 \% \mathrm{Cl}$ & $p$ value & HR & $95 \% \mathrm{Cl}$ & $p$ value \\
\hline Age $\geq 60$ years & 1.939 & $1.493-2.518$ & $<0.001$ & & & & 2.472 & $1.844-3.315$ & $<0.001$ \\
\hline IPSS-R higher-risk group & 3.397 & $2.436-4.736$ & $<0.001$ & & & & 3.047 & $2.159-4.300$ & $<0.001$ \\
\hline $\mathrm{ARC}<20 \times 10^{9} / \mathrm{L}$ & 2.685 & $2.045-3.525$ & $<0.001$ & 2.675 & $2.021-3.541$ & $<0.001$ & 2.118 & $1.563-2.870$ & $<0.001$ \\
\hline U2AF1 & 1.368 & $0.995-1.879$ & 0.053 & 1.407 & $1.018-1.945$ & 0.039 & & & \\
\hline SF3B1 & 1.081 & $0.702-1.664$ & 0.724 & & & & & & \\
\hline SRSF2 & 1.705 & $0.953-3.052$ & 0.072 & 2.229 & $1.228-4.048$ & 0.008 & & & \\
\hline TET2 & 1.844 & $1.217-2.795$ & 0.004 & 2.102 & $1.378-3.207$ & 0.001 & & & \\
\hline SETBP1 & 1.653 & $1.033-2.647$ & 0.036 & & & & 2.110 & $1.200-3.712$ & 0.010 \\
\hline TP53 & 2.915 & $1.967-4.320$ & $<0.001$ & 2.911 & $1.937-4.374$ & $<0.001$ & & & \\
\hline NRAS & 1.837 & $1.134-2.976$ & 0.014 & & & & & & \\
\hline PTPN11 & 2.481 & $1.350-4.559$ & 0.003 & 1.988 & $1.058-3.735$ & 0.033 & 2.206 & $1.108-4.390$ & 0.024 \\
\hline
\end{tabular}

$H R$ hazard ratio, $\mathrm{Cl}$ confidence interval. 
risk cohorts, patients with lower ARC had shorter OS than those with higher ARC $(p<0.001 ; p<0.001$; Fig. $4 \mathrm{~b}, \mathrm{c})$. The median OS of lower-risk patients with lower ARC was 22 months, which was shorter than that of lower-risk patients with higher ARC (not reached; $p<0.001$; Fig. 4d) and longer than higher-risk patients with lower ARC (12, 95\% CI, 10-14 months; $p=0.006$; Fig. 4d), while was similar to that of higher-risk and higher ARC patients (20, 95\% CI, 17-23 months; $p=0.370$; Fig. 4d).

Given that the BM hematopoietic activity declines with age, we then assessed aging effects by dividing all patients into younger ( $<60$ years) and older ( $\geq 60$ years) cohorts and analyzed the survival impact of ARC in each age group. As expected, patients with lower ARC had higher proportion of older patients compared with those with higher ARC (43.2\% vs. $32.8 \% ; p=0.014$; Table 1$)$. Nevertheless, regardless in younger or older cohort, patients with lower ARC had poorer OS than the others $(p<0.001 ; p<0.001$; Fig. 4e, f).

\section{Impact of ARC levels on OS under different treatment regimens}

To explore the effect of ARC on survival under different treatment regimens, we compared the OS of patients stratified by ARC who received distinct therapies. We found that among both IPSS-R lower-risk patients, who received cyclosporine (at an initial dose of $3 \mathrm{mg} / \mathrm{Kg} /$ day and modified based on blood concentration, hematologic responses and adverse effects) combined with danazol( \pm thalidomide), and higher-risk patients, who received decitabine therapy at the schedule of $20 \mathrm{mg} / \mathrm{m}^{2} /$ day for 5 days, every 28 days, patients with lower ARC had shorter OS compared with patients with higher ARC $(p<0.001 ; p<$ 0.001; Fig. 4g, h). Of note, among patients who received Allo-HSCT, no difference of OS was observed between patients with lower and higher ARC ( $p=0.525$; Fig. $4 \mathrm{i})$.

\section{Impact of different cut-off value of ARC on OS}

The above results showed that utilizing ARC of $20 \times$ $10^{9} / \mathrm{L}$ as cutoff could properly discriminate prognosis of MDS patients. Meanwhile, we adopted a bio-informatics tool X-tile ${ }^{28}$ to define an ARC threshold of $19.4 \times 10^{9} / \mathrm{L}$ to predict prognosis and obtained similar results to the cutoff of $20 \times 10^{9} / \mathrm{L}$ (data were not shown). We also used $\mathrm{X}$-tile to define three subgroups of patients with different outcomes taking 19.4 and $43 \times 10^{9} / \mathrm{L}$ as threshold. In general, as ARC became lower, the peripheral blood cell count, BM erythroblast percentage and numbers of CFUC became lower (Table S3, Fig. S10); the incidence of high-risk factors became higher, such as higher BM blast percentage, complex karyotype and several poor prognostic mutations (Table S3 and Figs. S11 and 12). In univariate analysis, patients with $\mathrm{ARC}<19.4 \times 10^{9} / \mathrm{L}$ or $19.4 \leq \mathrm{ARC}<43 \times 10^{9} / \mathrm{L}$ both showed poor OS compared with patients with $\mathrm{ARC} \geq 43 \times 10^{9} / \mathrm{L}$ (Table S4 and Fig. S13A). However, no difference of survival was found between patients with $19.4 \leq \mathrm{ARC}<43 \times 10^{9} / \mathrm{L}$ and patients with $\mathrm{ARC} \geq 43 \times 10^{9} / \mathrm{L}$ in multivariate analysis or subgroup analysis (Table S4 and Fig. S13B-H). While ARC $<19.4 \times 10^{9} / \mathrm{L}$ was still an independent poor prognostic factor (Table S4).

\section{Discussion}

In this study, by sorting HSCs and MEPs from MDS patients and inducing differentiation towards erythroid lineage ex vivo, we confirmed the impairments of erythropoietic activity in BM of MDS and, for the first time, found the precocity and premature aging occurring in MDS erythroid cells. To further determine prognostic implications in respect of the severity of ineffective erythropoiesis, we analyzed the clinical data in a large cohort of MDS patients and found that the severe ineffective erythropoiesis (defined by ARC $<20 \times 10^{9} / \mathrm{L}$ ) in MDS served as a powerful independent prognostic factor, which was associated with poor OS and compromised drug response.

The deficiency of erythroid lineage was proved to be most prominent in $\mathrm{MDS}^{4}$. Our prior study ${ }^{13}$ showed that numbers of BFU-E and CFU-E in MDS patients were obviously lower than healthy controls, which was confirmed in this study after expanding the sample size. In the present study, we further found that levels of HSCs and MEPs, which is a transitional stage of differentiation to both megakaryocytes and erythrocytes earlier than the BFU-E stage ${ }^{29,30}$, had already reduced in MDS. For a long time, the increased apoptosis of BM erythroid cells was considered to be responsible for ineffective erythropoiesis in MDS. Recently, numerous studies have yielded accumulating evidence about the contribution of pyroptosis, a caspase-1-dependent lytic cell death, and impaired terminal erythroid differentiation in the ineffective erythropoiesis of $\mathrm{MDS}^{8,9,31}$. In this study, after inducing the erythroid differentiation from patient-derived MEPs, we unexpectedly found the precocity of erythroid cells during differentiation, namely, the premature generation of hemoglobin at erythroid progenitor cells along with premature aging detected during erythropoiesis. Results of RNA-seq confirmed that aging pathways were upregulated progressively in MDS patients compared with normal controls, which was consistent with the phenotype discovered by functional experiments. Supposedly, such acceleration of the erythroid differentiation process may intent to compensate for the compromised erythropoiesis, which, however, even worsen erythropoietic disorders; or it may be parallel with other molecular events causing ineffective erythropoiesis from de novo. Definite mechanisms require further investigations.

All the data mentioned above implied that the defects of erythroid precursors, from both quantitative and 


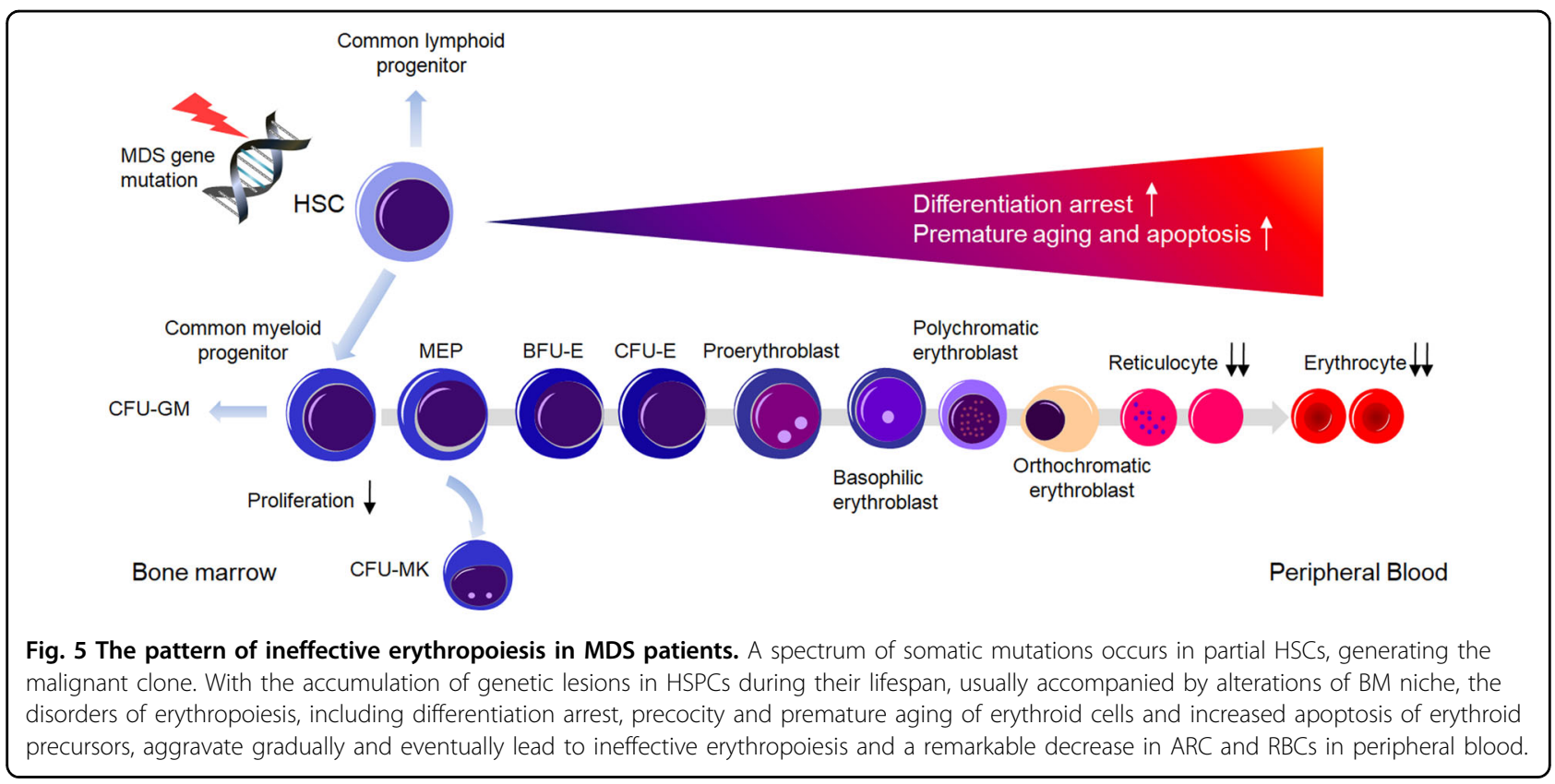

qualitative level, contributed to the impairment of erythropoietic activity in MDS. As shown in Fig. 5, a spectrum of somatic mutations occurred in partial HSCs, generating the malignant clone. With the accumulation of genetic lesions in HSPCs during their lifespan, usually accompanied by alterations in BM niche, the disorders of erythropoiesis, including differentiation arrest, precocity and premature aging of erythroid cells and then increased apoptosis of erythroid precursors, aggravate gradually and eventually lead to a remarkable decrease in ARC and RBCs.

Although the ineffective erythropoiesis is one of hallmarks of MDS, there is still no accurate biomarker to quantify it. ARC in peripheral blood is a reliable indicator for reflecting the erythropoietic activity of $\mathrm{BM}$ and thus maybe a useful biomarker for evaluating the severity of ineffective erythropoiesis. Using an ARC $<20 \times 10^{9} / \mathrm{L}$ as the cut-off, which was adopted from the definition criteria of SAA according to $\mathrm{BCSH}$, relationships of ineffective erythropoiesis to survival in MDS were determined. Our data showed that severe ineffective erythropoiesis defined by reduced ARC was an independent prognostic factor of inferior OS in MDS. Several variables should be responsible for this poor outcome. Firstly, the ineffective erythropoiesis is an essential feature of MDS and its degree could reflect the severity of the disease, for patients with more severe dyserythropoiesis may suffer more hits during the process of proliferation and differentiation; secondly, severe ineffective erythropoiesis is closely associated with some poor prognostic factors which has been enrolled in the IPSS- $R$ system, including higher $\mathrm{BM}$ blast percentage and more aggressive cytogenetic abnormalities; thirdly, it is correlated with several high-risk gene mutations, such as TP53 and ZRSR2 ${ }^{32-36}$.

The immunosuppressive treatment is a fundamental therapy for lower-risk $\mathrm{MDS}^{37}$. In recent years, hypomethylating agents are widely used for higher-risk MDS and significantly improve the prognosis of these patients ${ }^{38,39}$. However, these drugs are not curative therapy for MDS and their effects on survival to some of patients are rather limited. To date, allo-HSCT remains the only treatment choice for a possible cure of $\mathrm{MDS}^{40}$. Our primary results showed that the poor outcome of severe reduced ARC could not be reversed by the immunosuppressive or hypo-methylating agents. Also, lower ARC was significantly associated with higher EPO level, which was considered as a biomarker for poor erythroid response to erythropoiesis stimulating agents ${ }^{41}$ Given that the severe ineffective erythropoiesis did not affect survival after HSCT, early HSCT should be suitable therapy for these patients.

There are several limitations to our study. For example, as MDS cells are notoriously difficult to be isolated and cultured ex vivo, although our limited cellular studies enable to provide mechanistic insights of precocity and premature aging regarding ineffective erythropoiesis in MDS, future investigation with larger cohort of patients would warrant thoroughly deciphering its underlying detailed molecular mechanisms.

In conclusion, precocity and premature aging during erythroid differentiation is one of profound defects of erythroid precursors in MDS contributing to the ineffective erythropoiesis. The severe ineffective erythropoiesis defined by $\mathrm{ARC}<20 \times 10^{9} / \mathrm{L}$ is a powerful independent 
prognostic factor for inferior OS in MDS. Patients with lower ARC could not benefit from immunosuppressive treatment and hypo-methylating agents, wherefore should be candidates for Allo-HSCT.

\section{Acknowledgements}

We are supported by National Natural Science Funds (No. 81870104, No. 81530008 , No. 81470295, No. 81770129, No. 81470297, No. 81870089), Tianjin Key Natural Science Funds(18JCZDJC34900), Tianjin Municipal Science and Technology Commission Grant (15JCYBJC54500), CAMS Initiative Fund for Medical Sciences(No. 2016-12M-1-001, No. 2016-I2M-3-002, No. 2016-I2M-1-018 and No. 2017-I2M-1-015), the National Key Research and Development Program of China (2016YFA0102300, 2017YFA0103100 and 2017YFA0103102), Non-profit Central Research Institute Fund of Chinese Academy of Medical Sciences (2019PT310022), the Fundamental Research Funds for the Central Universities (3332018157).

\section{Author details}

${ }^{1}$ MDS and MPN Centre, Institute of Hematology and Blood Diseases Hospital, Chinese Academy of Medical Sciences \& Peking Union Medical College, Tianjin, China. ${ }^{2}$ State Key Laboratory of Experimental Hematology, Institute of Hematology and Blood Diseases Hospital, Chinese Academy of Medical Sciences \& Peking Union Medical College, Tianjin, China. ${ }^{3}$ National Clinical Research Centre for Blood Diseases, Institute of Hematology and Blood Diseases Hospital, Chinese Academy of Medical Sciences \& Peking Union Medical College, Tianjin, China. ${ }^{4}$ Pathology Centre, Institute of Hematology and Blood Diseases Hospital, Chinese Academy of Medical Sciences \& Peking Union Medical College, Tianjin, China. ${ }^{5}$ Haematology Section, Division of Experimental Medicine, Department of Medicine, Imperial College London, London, UK. ${ }^{6}$ Divisions of Pathology and Experimental Hematology and Cancer Biology, Cincinnati Children's Hospital Medical Center, Cincinnati, OH, USA

\section{Author contributions}

Z.J.X. and L.H.S. designed the research, was the principal investigator, and took primary responsibility for the paper; Z.J.X., L.H.S., H.J.H., C.L.X., J.G., S.R.R., and J.Q.L. acquired the data, analyzed and interpreted the data, performed statistical analysis and drafted the article; H.J.H., B.L.,Y.D.Z., and M.J. acquired clinical data; Z.J.X., T.J.Q., Z.F.X., B.L., M.J., S.Q.Q., L.J.P., and N.B.H. recruited the patients; Z.J.X., L.H.S., H.J.H., C.L.X.G.H., and R.P.G. prepared the paper.

\section{Conflict of interest}

R.P.G. is a part-time employee of Celgene Corp. The remaining authors declare that they have no conflict of interest.

\section{Publisher's note}

Springer Nature remains neutral with regard to jurisdictional claims in published maps and institutional affiliations.

Supplementary Information accompanies this paper at (https://doi.org/ 10.1038/s41408-020-00349-4).

Received: 23 April 2020 Revised: 29 July 2020 Accepted: 3 August 2020 Published online: 14 August 2020

\section{References}

1. Elias, H. K. et al. Stem cell origin of myelodysplastic syndromes. Oncogene 33, 5139-5150 (2014)

2. Bejar, R., Levine, R. \& Ebert, B. L. Unraveling the molecular pathophysiology of myelodysplastic syndromes. J. Clin. Oncol. 29, 504-515 (2011).

3. Tefferi, A. \& Vardiman, J. W. Myelodysplastic syndromes. N. Engl. J. Med. 361, 1872-1885 (2009).

4. Flores-Figueroa, E., Gutierrez-Espindola, G., Guerrero-Rivera, S., Pizzuto-Chavez, J. \& Mayani, H. Hematopoietic progenitor cells from patients with myelodysplastic syndromes: in vitro colony growth and long-term proliferation. Leuk. Res. 23, 385-394 (1999).

5. Maratheftis, C. I., Andreakos, E., Moutsopoulos, H. M. \& Voulgarelis, M. Toll-like receptor-4 is up-regulated in hematopoietic progenitor cells and contributes to increased apoptosis in myelodysplastic syndromes. Clin. Cancer Res. 13 1154-1160 (2007)

6. Chen, $X$. et al. Induction of myelodysplasia by myeloid-derived suppressor cells. J. Clin. Invest. 123, 4595-4611 (2013).

7. Frisan, E. et al. Defective nuclear localization of Hsp70 is associated with dyserythropoiesis and GATA-1 cleavage in myelodysplastic syndromes. Blood 119, 1532-1542 (2012).

8. Sallman, D. A. \& List, A. The central role of inflammatory signaling in the pathogenesis of myelodysplastic syndromes. Blood 133, 1039-1048 (2019).

9. Basiorka, A. A. et al. The NLRP3 inflammasome functions as a driver of the myelodysplastic syndrome phenotype. Blood 128, 2960-2975 (2016).

10. Piva, E., Brugnara, C., Spolaore, F. \& Plebani, M. Clinical utility of reticulocyte parameters. Clin. Lab. Med. 35, 133-163 (2015).

11. Marsh, J. C. et al. Guidelines for the diagnosis and management of aplastic anaemia. Br. J. Haematol. 147, 43-70 (2009).

12. Arber, D. A. et al. The 2016 revision to the World Health Organization classification of myeloid neoplasms and acute leukemia. Blood 127, 2391-2405 (2016).

13. Li, B. et al. Colony-forming unit cell (CFU-C) assays at diagnosis: CFU-G/M cluster predicts overall survival in myelodysplastic syndrome patients independently of IPSS-R. Oncotarget 7, 68023-68032 (2016).

14. Li, B. et al. The usefulness of mutational data on prognosis of myelodysplastic syndromes: alone or incorporated into the IPSS-R? Br. J. Haematol. 183, 815-819 (2018)

15. Greenberg, P. L. et al. Revised international prognostic scoring system for myelodysplastic syndromes. Blood 120, 2454-2465 (2012).

16. Giagounidis, A. Current treatment algorithm for the management of lower-risk MDS. Hematology Am. Soc. Hematol. Educ. Program 2017, 453-459 (2017).

17. Maki, $K$. et al. Expressional changes of genes and miRNA in common megakaryocyte-erythroid progenitors from lower-risk myelodysplastic syndrome. Int J. Hematol. 100, 361-369 (2014).

18. Liu, J. et al. Long non-coding RNA-dependent mechanism to regulate heme biosynthesis and erythrocyte development. Nat. Commun. 9, 4386 (2018).

19. Orkin, S. H., Harosi, F. I. \& Leder, P. Differentiation in erythroleukemic cells and their somatic hybrids. Proc. Natl Acad. Sci. USA 72, 98-102 (1975).

20. Li, L. et al. [Preliminary study of diagnosis of patients with myelodysplastic syndromes by routine laboratory parameters]. Zhonghua Xue Ye Xue Za Zhi 29 , 623-628 (2008).

21. Sepe, S. et al. Inefficient DNA repair is an aging-related modifier of Parkinson's disease. Cell Rep 15, 1866-1875 (2016).

22. Schermer, B. et al. Transcriptional profiling reveals progeroid Ercc1(-/Delta) mice as a model system for glomerular aging. BMC Genomics 14, 559 (2013).

23. Satoh, J., Kawana, N. \& Yamamoto, Y. Pathway analysis of ChIP-Seq-based NRF1 target genes suggests a logical hypothesis of their involvement in the pathogenesis of neurodegenerative diseases. Gene Regul. Syst. Bio. 7, 139-152 (2013).

24. Lin, C. H., Lin, E. \& Lane, H. Y. Genetic biomarkers on age-related cognitive decline. Front. Psychiatry 8, 247 (2017).

25. Fontenay-Roupie, $M$. et al. Ineffective erythropoiesis in myelodysplastic syndromes: correlation with Fas expression but not with lack of erythropoietin receptor signal transduction. Br. J. Haematol. 106, 464-473 (1999).

26. Xiong, X. X. et al. Piperlongumine induces apoptotic and autophagic death of the primary myeloid leukemia cells from patients via activation of ROS-p38/ JNK pathways. Acta Pharmacol. Sin. 36, 362-374 (2015).

27. Steensma, D. P. \& Tefferi, A. The myelodysplastic syndrome(s): a perspective and review highlighting current controversies. Leuk Res. 27, 95-120 (2003).

28. Camp, R. L., Dolled-Filhart, M. \& Rimm, D. L. X-tile: a new bio-informatics tool for biomarker assessment and outcome-based cut-point optimization. Clin. Cancer Res. 10, 7252-7259 (2004).

29. Velten, L. et al. Human haematopoietic stem cell lineage commitment is a continuous process. Nat. Cell Biol. 19, 271-281 (2017).

30. Xavier-Ferrucio, J. \& Krause, D. S. Concise review: bipotent megakaryocyticerythroid progenitors: concepts and controversies. Stem Cells 36, 1138-1145 (2018).

31. Ali, A. M. et al. Severely impaired terminal erythroid differentiation as an independent prognostic marker in myelodysplastic syndromes. Blood Adv. 2, 1393-1402 (2018).

32. Papaemmanuil, E. et al. Clinical and biological implications of driver mutations in myelodysplastic syndromes. Blood 122, 3616-3627 (2013).

33. Bejar, R. et al. Clinical effect of point mutations in myelodysplastic syndromes. N. Engl. J. Med. 364, 2496-2506 (2011). 
34. Tefferi, A. et al. Targeted next-generation sequencing in myelodysplastic syndromes and prognostic interaction between mutations and IPSS-R. Am. J. Hematol. 92, 1311-1317 (2017).

35. Makishima, $\mathrm{H}$. et al. Dynamics of clonal evolution in myelodysplastic syndromes. Nat. Genet. 49, 204-212 (2017).

36. Gangat, N. et al. Mutations and prognosis in myelodysplastic syndromes: karyotype-adjusted analysis of targeted sequencing in 300 consecutive cases and development of a genetic risk model. Am. J. Hematol. 93, 691-697 (2018),

37. Parikh, A. R., Olnes, M. J. \& Barrett, A. J. Immunomodulatory treatment of myelodysplastic syndromes: antithymocyte globulin, cyclosporine, and alemtuzumab. Semin. Hematol. 49, 304-311 (2012).
38. Kantarjian, $\mathrm{H}$. et al. Decitabine improves patient outcomes in myelodysplastic syndromes: results of a phase III randomized study. Cancer Am. Cancer Soc. 106, 1794-1803 (2006).

39. Silverman, L. R. et al. Randomized controlled trial of azacitidine in patients with the myelodysplastic syndrome: a study of the cancer and leukemia group B. J. Clin. Oncol. 20, 2429-2440 (2002).

40. Gangat, N., Patnaik, M. M. \& Tefferi, A. Myelodysplastic syndromes: Contemporary review and how we treat. Am. J. Hematol. 91, 76-89 (2016).

41. Houston, B. L. et al. A predictive model of response to erythropoietin stimulating agents in myelodysplastic syndrome: from the Canadian MDS patient registry. Ann. Hematol. 96, 2025-2029 (2017). 